\title{
Development of an Analytical Method for Arbitrary Shaped Pattern Synthesis of Planar Arrays
}

\author{
Mahdi BOOZARI ${ }^{1}$, Mohammad KHALAJ-AMIRHOSSEINI ${ }^{2}$ \\ ${ }^{1}$ Dept. of Electrical Engineering, Ferdowsi University of Mashhad, Azadi, Mashhad, Iran \\ ${ }^{2}$ School of Electrical Engineering, Iran University of Science and Technology, Resalat, Tehran, Iran \\ mahdiboozari@yahoo.com, khalaja@iust.ac.ir \\ Submitted June 12, 2021 / Accepted November 2, 2021
}

\begin{abstract}
In practice, it is often necessary to design an array that will yield desired radiation pattern. For this purpose, several time-consuming algorithms are introduced in the literature. In this paper, an analytical method is presented to synthesize the radiation pattern of planar and ring arrays. In this method, two new parameters are defined to reconstruct the array factor and simplify the calculation complexity. To accomplish this, we use the double integral to generate two distinct Sinc functions from a bivariate function utilizing the sampling theory notion. This stage generates a set of linear equations that, when solved, yields the complex excitation coefficients. The proposed method is verified by presenting several practical examples. Also, the performance of the method is compared with that of other approaches. The results show that the proposed method is a good candidate for synthesizing a prescribed pattern of planar arrays.
\end{abstract}

\section{Keywords}

Array factor, integration, least square method, pattern synthesizing

\section{Introduction}

The array of antennas has many advantages, including high gain and capability of the spatial scan. These features make them suitable for a variety of applications such as radar tracking and 5G communications [1-6]. Planar arrays are capable of searching the space in spherical coordinates in both the elevation and azimuth directions. The radiation pattern of a planar array is more symmetrical and has the lower side lobes than linear arrays [7], [8].

Until now, several methods have been introduced for the synthesis of radiation pattern of a linear and planar array. These methods are classified into two groups. The first category consists of analytical techniques such as the Fast Fourier transform (FFT) [9], Deterministic Approach [10], Deterministic Space Tapering Technique [11], statistical techniques and least square method [12]. The second type is based on classical or evolutionary techniques like Genetic Algorithm (GA), Particle Swarm Optimization (PSO), and Differential Evolution Algorithm (DEA) [13]. The type of antennas used in the array must be known in order to consider the mutual coupling effect. By knowing the elements of an array, the mutual coupling effect can be considered by approaches such as numerical methods and full-wave simulations [14].

Due to the complexity of the designing process of a planar array, the synthesizing methods introduced in the literature are primarily based on numerical and algorithmbased techniques, and the analytical methods are scarce in the literature. The existing analytical techniques are only applicable to particular types of planar arrays. For example, closed-form formulas are available only for ring arrays with Taylor and Bayliss distribution [15], [16].

An analytical approach is described in this paper that may be used to synthesize the radiation pattern of planar and circular arrays in general. Other works, on the other hand, are algorithm-based or use iterative techniques. It is clear that the non-analytical methods have low efficiency. Additionally, the analytical approaches provide a clear relationship between the input and output parameters of the problem, which allow intelligent modifications. In this method, it is tried to utilize all the data of the prescribed pattern. The target is achieved by defining two new parameters and using the dual integral operator over the specified intervals. In other words, the integral operator allocates the known values to unknown functions. Two distinct Sinc functions are formed as a result of the integrating step, which inspire the sampling theory concept from a bivariate function. A set of linear equations is created by selecting different intervals, in which the solution of it provides the complex excitation coefficients of array elements.

\section{Theory of the Proposed Method}

Figure 1 shows the geometry of a planar array consisting of isotropic elements placed in the $x-y$ plane. In this case, the array factor can be written as [15]. 


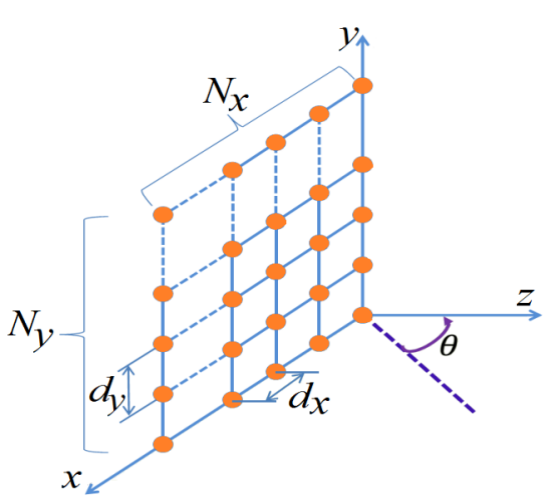

Fig. 1. The geometry of a planar array.

$$
\begin{gathered}
F=\sum_{r=1}^{N_{\mathrm{x}}} \sum_{t=1}^{N_{\mathrm{y}}} I_{r t} \exp \left(\mathrm{j} k x_{r} u\right) \exp \left(\mathrm{j} k y_{t} v\right), \\
u=\sin \theta \cos \varphi, v=\sin \theta \sin \varphi \\
x_{r}=r d_{\mathrm{x}}, y_{t}=t d_{\mathrm{y}}
\end{gathered}
$$

where $k=2 \pi / \lambda, I_{r t}, \theta$ and $\varphi$ are the wave number, the relative excitation of $r t^{\text {th }}$ element, elevation and azimuth angles, respectively. Also, $d_{\mathrm{x}}, d_{\mathrm{y}}, N_{\mathrm{x}}$ and $N_{\mathrm{y}}$ show the distance between the elements along the $\mathrm{x}, \mathrm{y}$-axis, and the number of array elements in the $\mathrm{x}, \mathrm{y}$ directions, respectively. Since the elevation and azimuth angles change from 0 to $\pi$ and 0 to $2 \pi$, respectively, both the parameters $u$ and $v$ change from -1 to +1 .

Integrating the left and right sides of (1) over the symmetrical intervals $-u_{m} \leq u \leq u_{m}$ and $-v_{p} \leq v \leq v_{p}$ leads to [17], [18]:

$$
\begin{gathered}
g=\int_{-v_{p}}^{+v_{p}+u_{m}} \int_{u_{m}} F \mathrm{~d} u \mathrm{~d} v, \\
h=\sum_{r=1}^{N x} \sum_{t=1}^{N y} 4 u_{m} v_{p} I_{r t} F_{\mathrm{S}}, \\
F_{\mathrm{S}}=\frac{\sin \left(k x_{r} u_{m}\right)}{k x_{r} u_{m}} \frac{\sin \left(k y_{t} v_{p}\right)}{k y_{t} v_{p}} .
\end{gathered}
$$

We know from sampling theory that a bivariate function can be reconstructed using two separated Sinc functions. It is worth noting that the Sinc function plays the role of the sampling function $F_{\mathrm{S}}\left(u_{m}, v_{p}\right)$ and the reconstructed process is done from only restricted samples of itself lying within a predefined interval [19]. The obtained equations (4), (5) confirm the mentioned subject. The symmetrical integration intervals in (3) for $m=1,2, \ldots, M_{\mathrm{u}}$, $p=1,2, \ldots, P_{\mathrm{v}}$ are as follows

$$
\begin{aligned}
& {\left[\begin{array}{ll}
-u_{m} & u_{m}
\end{array}\right]=\left[\begin{array}{ll}
-m \Delta u & m \Delta u
\end{array}\right]} \\
& {\left[\begin{array}{ll}
-v_{p} & v_{p}
\end{array}\right]=\left[\begin{array}{ll}
-p \Delta v & p \Delta v
\end{array}\right] .}
\end{aligned}
$$

Since parameters $u, v$ change from -1 to +1 , parameters $\Delta u$ and $\Delta v$ can be defined as [17]

$$
\Delta u=\frac{2 \pi}{M_{\mathrm{u}}}, \Delta v=\frac{2 \pi}{P_{\mathrm{v}}}
$$

in which $M_{\mathrm{u}}$ and $P_{\mathrm{v}}$ are the number of integrating intervals or the total number of samples for $u$ and $v$ variables, respectively. It is seen that equations (3) to (5) lead to a system of linear equations, the solution of which is the array's excitation coefficients [20]:

$$
\begin{aligned}
& \mathbf{A X}=\mathbf{B}, \\
& \mathbf{A}=[\bar{h}]_{m p \times n}, \bar{h}=4\left(\frac{2 m \pi}{M_{\mathrm{u}}}\right)\left(\frac{2 p \pi}{P_{\mathrm{v}}}\right) F_{\mathrm{S}}, \\
& F_{\mathrm{S}}=\operatorname{sinc}\left(\frac{2 m \pi k x_{r}}{M_{\mathrm{u}}}\right) \operatorname{sinc}\left(\frac{2 p \pi k y_{t}}{P_{\mathrm{v}}}\right) \text {, } \\
& \mathbf{B}=\left[g(1,1) \ldots g(m, p) \ldots g\left(M_{\mathrm{u}}, P_{\mathrm{v}}\right)\right]^{\mathrm{T}}, \\
& \mathbf{X}=\left[\begin{array}{llll}
\mathbf{X}_{1} & \mathbf{X}_{i} & \cdots & \mathbf{X}_{N_{\mathrm{x}}}
\end{array}\right]^{\mathrm{T}}, \\
& \mathbf{X}_{i}=\left[\begin{array}{lll}
I_{i 1} & \cdots & I_{i N_{\mathrm{y}}}
\end{array}\right]^{\mathrm{T}}, i=1, \ldots, N_{\mathrm{x}}
\end{aligned}
$$

where $\mathbf{X}$ and $\mathbf{B}$ are vectors including the excitation coefficients of array elements and the integration results of the desired pattern, respectively. Additionally, matrix A contains the samples of Sinc functions, as illustrated in (5). Because matrix $\mathbf{A}$ is left-invertible for all practical arrays, equation (9) can be solved using the Pseudo-Inverse (PI) method. After applying the PI method, the unknowns of (9) are determined as follows [21]

$$
\mathbf{X}=\left(\mathbf{A}^{\mathrm{T}} \mathbf{A}\right)^{-1} \mathbf{A}^{\mathrm{T}} \mathbf{B}
$$

It is seen from (10) that all elements of matrix $\mathbf{A}$ are only dependent on the location of the array elements $\left(x_{r}, y_{t}\right)$ and discrete variables $r$ and $t$. In other words, matrix $\mathbf{A}$ is independent of oscillatory variables $u$ and $v$. It is worth noting that the variables $u$ and $v$ are inherently vulnerable to noise. In other words, the integral operator has protected the proposed synthesis procedure from undesired changes. On the other hand, matrix A only includes the real values. Hence, the proposed method will efficiently decrease the computational time and error.

Because the integration process utilizes all of the data associated with the desired pattern, data loss is negligible in the suggested method. This property distinguishes the method from other sampling-based methods, such as the Woodward-Lawson method, which suffers from a lack of local control over the desired pattern's unshaped area [7].

The only requirement for the proposed technique, according to (3), is that the desired array factor be integrable throughout the integration interval and piecewise continuous [22]. In other words, the desired array factor must satisfy the Dirichlet condition for the proposed method to be convergent, as follows [23]

$$
\iint_{u, v}|F| \mathrm{d} u \mathrm{~d} v<\infty .
$$


The Dirichlet condition (16) is usually satisfied in practical arrays, so the proposed method is general. The parameters $M_{\mathrm{u}}$ and $P_{\mathrm{v}}$ can also be determined using the Nyquist theorem, as shown below [18]

$$
M_{\mathrm{u}} \geq \frac{4\left(N_{\mathrm{x}}-1\right) d_{\mathrm{x}}}{\lambda}, P_{\mathrm{v}} \geq \frac{4\left(N_{\mathrm{y}}-1\right) d_{\mathrm{y}}}{\lambda} .
$$

Our studies show that for practical arrays, $M_{\mathrm{u}} \approx 5\left(N_{\mathrm{x}}-1\right) d_{\mathrm{x}} / \lambda$ and $P_{\mathrm{v}} \approx 5\left(N_{\mathrm{y}}-1\right) d_{\mathrm{y}} / \lambda$, are sufficient and the iterative process is not needed [21]. Because the number of $N_{\mathrm{x}}$ and $N_{\mathrm{y}}$ in symmetrical arrays is halved, just onehalf of the parameters $M_{\mathrm{u}}$ and $P_{\mathrm{v}}$ are required. In this case, we are faced with a real array factor as follows

$$
\begin{aligned}
& F=\sum_{r=1}^{N_{\mathrm{x}} / 2} \sum_{t=1}^{N_{\mathrm{y}} / 2} 2 I_{r t} F_{\mathrm{uv}}, N_{\mathrm{x}}, N_{\mathrm{y}}: \text { even }, \\
& F=I_{00}+\sum_{r=1}^{\frac{N_{\mathrm{x}}-1}{2}} \sum_{t=1}^{\frac{N_{\mathrm{y}}-1}{2}} 2 I_{r t} F_{\mathrm{uv}}, N_{\mathrm{x}}, N_{\mathrm{y}}: \text { odd }, \\
& F_{\text {uv }}=\cos \left(k x_{r} u\right) \cos \left(k y_{t} v\right)
\end{aligned}
$$

in which $I_{00}$ presents the excitation coefficient of the element placed in the array plane's center. Hence, for the symmetrical arrays, equation (4) is rewritten as follows

$$
\begin{gathered}
h=\sum_{r=1}^{N_{\mathrm{x}}} \sum_{t=1}^{N_{\mathrm{y}}} 4 u_{m} v_{p} I_{r t} F_{\mathrm{S}}, N_{\mathrm{x}}, N_{\mathrm{y}}: \text { even }, \\
h=4 I_{00} u_{\mathrm{m}} v_{\mathrm{p}}+\sum_{r=1}^{\frac{N_{\mathrm{x}}-1}{2}} \frac{N_{\mathrm{y}}-1}{\sum_{t=1}^{2}} 4 u_{m} v_{p} I_{r t} F_{\mathrm{S}}, N_{\mathrm{x}}, N_{\mathrm{y}}: \text { odd } .
\end{gathered}
$$

Function $F_{\mathrm{S}}$ is the same as defined in (5). Other synthesizing steps for symmetrical arrays are the same as previously stated.

The array factor for a planar array with elements arranged on a circular sheet in the $\mathrm{x}-\mathrm{y}$ plane is shown below [24]

$$
F=\sum_{n=1}^{N} I_{n} \exp \left(\mathrm{j} k R_{n} \sin \theta \cos \left(\varphi-\varphi_{n}\right)\right)
$$

in which $I_{n}$ is the complex excitation of the $n^{\text {th }}$ element and the pairs $\left(R_{n}, \varphi_{n}\right)$ show the position of the $n^{\text {th }}$ element in cylindrical coordinate. Equation (20) can be rewritten as follows in consideration of $(2 a)$ :

$$
F=\sum_{n=1}^{N} I_{n} \exp \left(\mathrm{j} R_{n} u \cos \varphi_{n}\right) \exp \left(\mathrm{j} R_{n} v \sin \varphi_{n}\right) .
$$

In this scenario and according to the approach outlined above, equation (4) should stay unchanged; however, equations (5) and (11) should be updated as follows:

$$
F_{\mathrm{S}}=\frac{\sin \left(R_{n} u_{m} \cos \varphi_{n}\right)}{R_{n} u_{m} \cos \varphi_{n}} \frac{\sin \left(R_{n} v_{p} \sin \varphi_{n}\right)}{R_{n} v_{p} \sin \varphi_{n}},
$$

$$
F_{\mathrm{S}}=\operatorname{sinc}\left(\frac{2 R_{n} \cos \varphi_{n}}{\left(M_{\mathrm{u}} / m \pi\right)}\right) \operatorname{sinc}\left(\frac{2 R_{n} \sin \varphi_{n}}{\left(P_{\mathrm{v}} / p \pi\right)}\right) .
$$

Equations (21) to (23) can be used for a circular ring array by substituting $R_{n}$ to $R_{0}$, where $R_{0}$ represents the ring radius. The array factor is independent of azimuth angle for a ring array with a large radius, and the array factor can be simplified as follows

$$
F=\sum_{n=1}^{N} I_{n} \exp \left(\mathrm{j} k R_{0} \sin \theta \cos \varphi_{n}\right) .
$$

In this case, we can define $u=k R_{0} \sin \theta$. Hence, equation (5) changes as

$$
F_{\mathrm{S}}=\operatorname{sinc}\left(\frac{2 m \pi \cos \varphi_{n}}{M_{\mathrm{u}}}\right)
$$

A radiation pattern with ring type side lobe levels is sometimes required. In this case, the main lobe has the same beam-width in all radiation planes. A linear array with transformations, as shown in [19], can be used to generate the desired radiation pattern of an array with ring type side lobes.

\section{Results Verification}

In this section, to verify the performance of the introduced method, several practical arrays are examined. The process of determining the analytical expression of all examples is available in the literature [15], [24].

\subsection{Example 1}

In the first example, an $8 \times 8$ Tschebyscheff array with ring type side lobes is considered. The distance between the elements in the $x$ and $y$ directions is equal to $0.5 \lambda$. The side lobe level is about $-25 \mathrm{~dB}$, and $M_{\mathrm{u}}=P_{\mathrm{v}}=16$. Figure 2 depicts the synthesized array's 3D radiation pattern. Figure 3 shows the radiation pattern in the u-v plane. In Fig. 4, the excitation coefficients are shown against the element locations in the $x-y$ plane, where all excitation coefficients have real values.

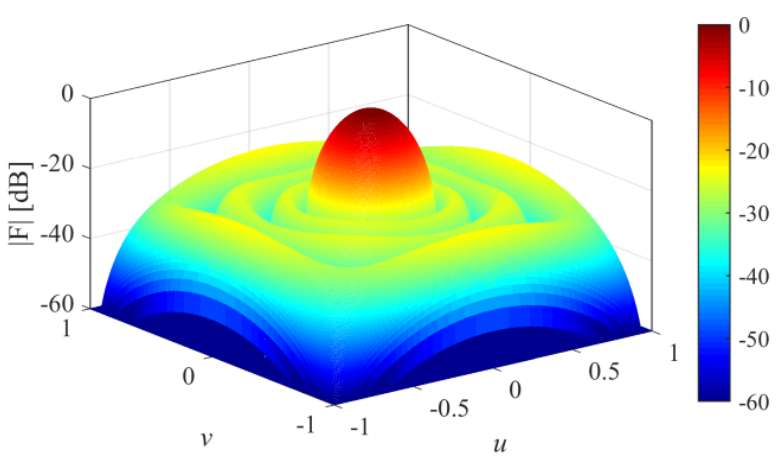

Fig. 2. The synthesized results of $8 \times 8$ Tschebyscheff array. 


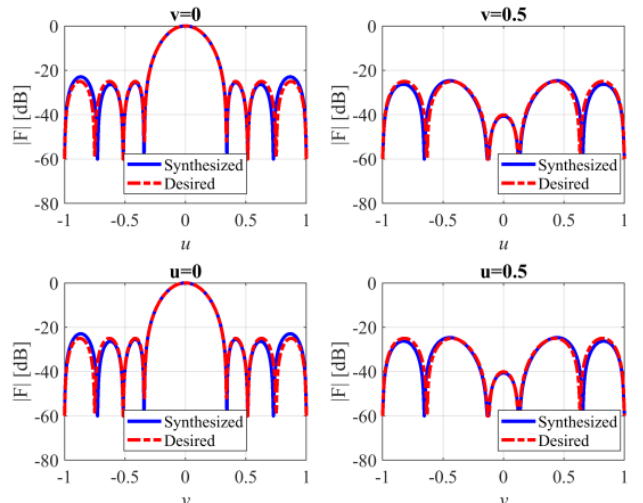

Fig. 3. Radiation pattern of Tschebyscheff array in u-v plane.

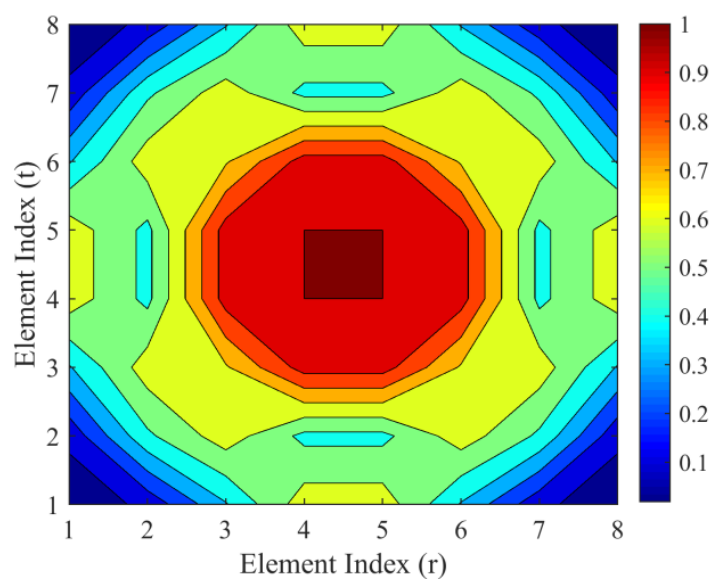

Fig. 4. The excitation coefficients of $8 \times 8$ Tschebyscheff array.

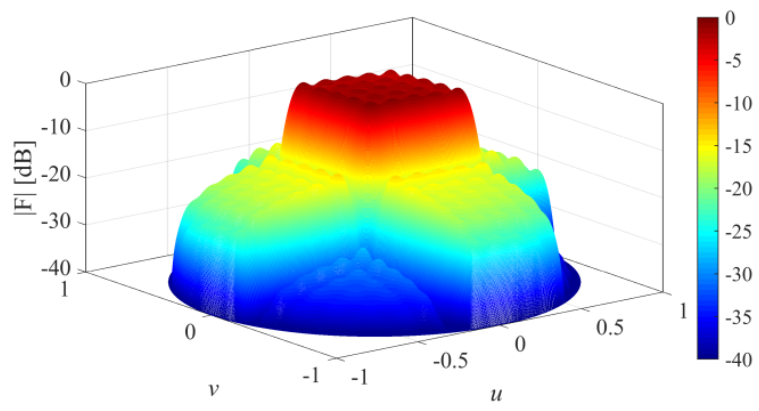

Fig. 5. The $3 D$ results of $30 \times 30$ array with flat-top pattern.
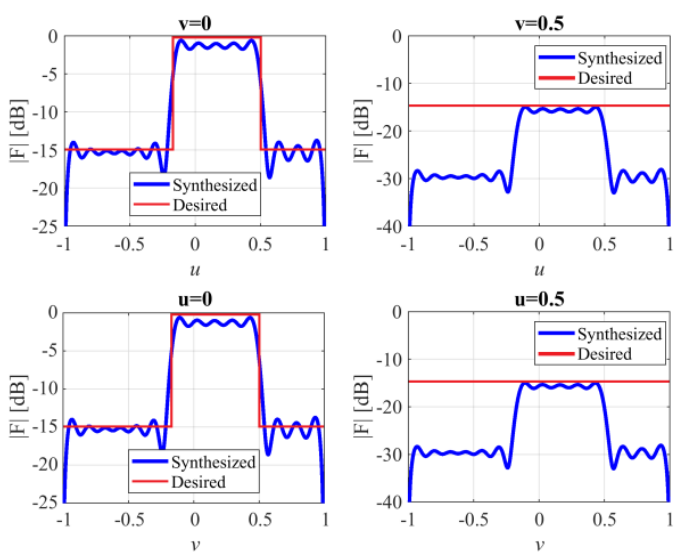

Fig. 6. Radiation pattern of flat-top array in u-v plane.

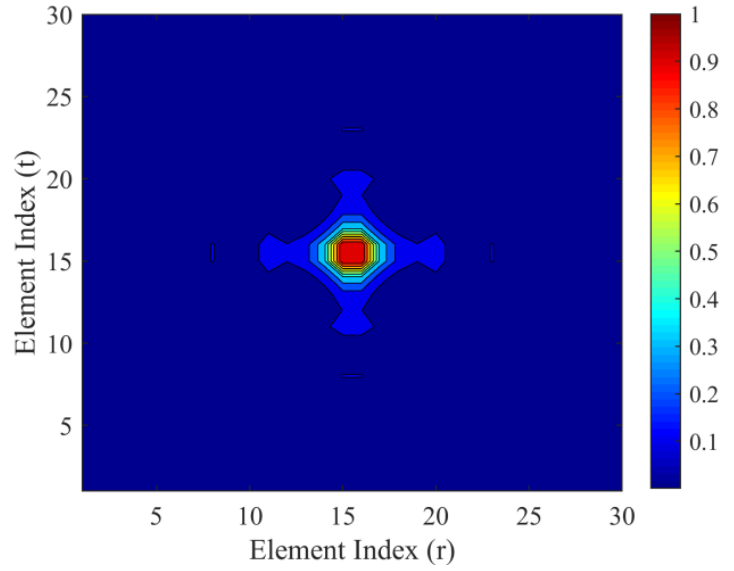

Fig. 7. The magnitude of the excitation currents of all elements of flat-top array.

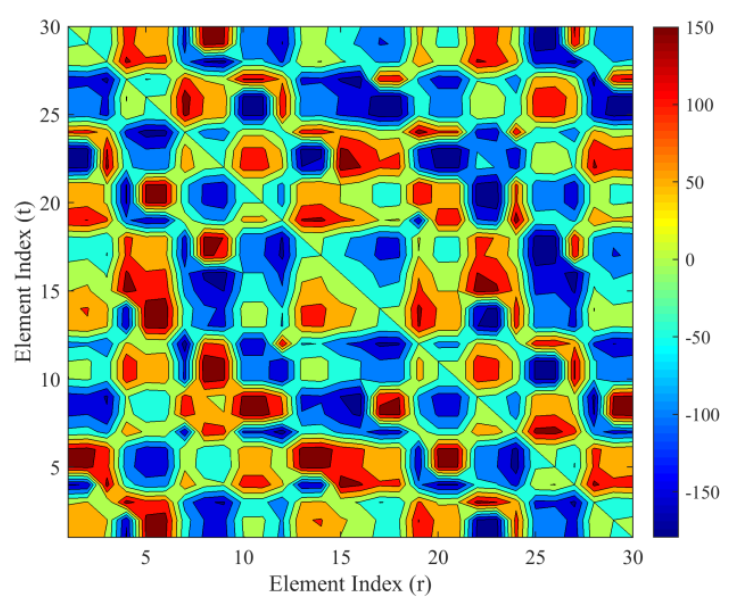

Fig. 8. The phase (in degree) of the excitation currents of all elements of flat-top array.

\subsection{Example 2}

The second example considers a symmetrical array with a flat-top radiation pattern. The designing parameters are $N_{\mathrm{x}}=N_{\mathrm{y}}=30, d_{\mathrm{x}}=d_{\mathrm{y}}=0.5 \lambda$ and $M_{\mathrm{u}}=P_{\mathrm{v}}=70$. Except for the main lobe angles, it is assumed that the normalized amplitude pattern is equal to or less than 0.2. Figures 5 and 6 show the obtained results in 3D format and in the u-v plane, respectively. The magnitude and phase (in degree) of all elements' excitation currents are also shown in Fig. 7 and 8 , respectively. According to Fig. 8 , the phase of the excitation currents is more critical than their magnitude.

\subsection{Example 3}

In some applications, and due to the interference effect, a planar array with two different values of side lobes is needed. In [21], an algorithm-based method for synthesis of the defined pattern is introduced. In the third example, the described pattern is investigated. To this end, a planar array with $N_{\mathrm{x}}=N_{\mathrm{y}}=21, d_{\mathrm{x}}=d_{\mathrm{y}}=0.5 \lambda$, and $M_{\mathrm{u}}=P_{\mathrm{v}}=40$ is considered. The first and second side lobe levels of the prescribed pattern are about $-16 \mathrm{~dB}$ and $-40 \mathrm{~dB}$, respectively. The obtained results in $3 \mathrm{D}$ format and in the $\mathrm{u}-\mathrm{v}$ 
plane are depicted in Fig. 9, 10, respectively. Figure 10 shows that the deep nulls for all value of azimuth angles are met very well. Figure 11 shows the under-studying array's excitation coefficients. The phase of all elements' excitation currents is zeros.

\subsection{Example 4}

In the fourth example, a planar array with a difference-type radiation pattern is regarded. The side lobe levels of the desired pattern are about -20 and $-25 \mathrm{~dB}$, respectively. The designing parameters are $N_{\mathrm{x}}=15, N_{\mathrm{y}}=10$, $d_{\mathrm{x}}=0.5 \lambda, d_{\mathrm{y}}=0.75 \lambda$. The obtained $3 \mathrm{D}$ radiation pattern is

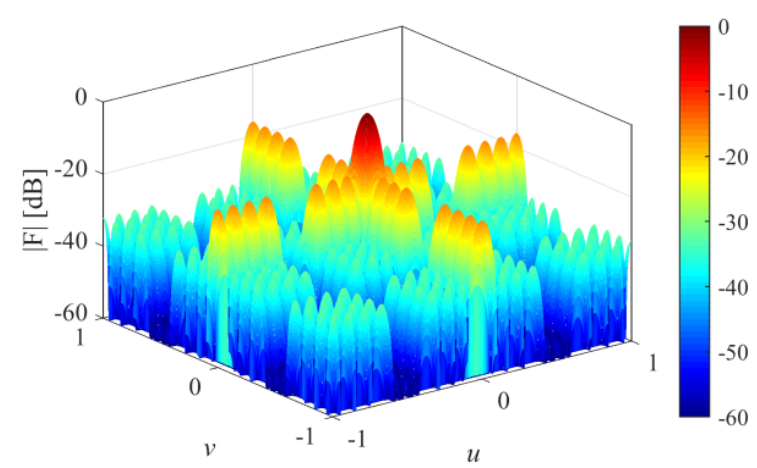

Fig. 9. The synthesized results of $21 \times 21$ array with two level side lobes.
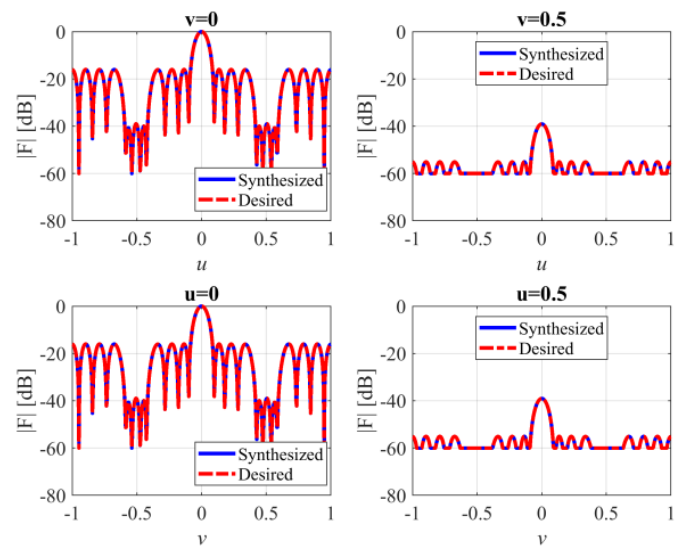

Fig. 10. Radiation pattern of the array with two level side lobes in u-v plane.

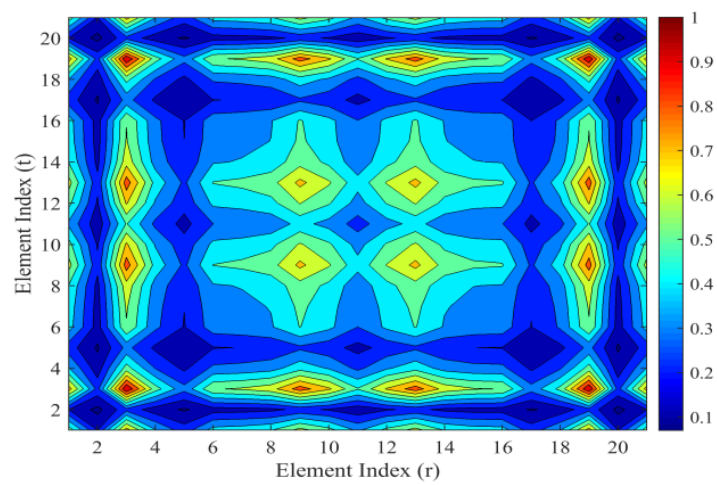

Fig. 11. The excitation currents of the array with two level side lobes.

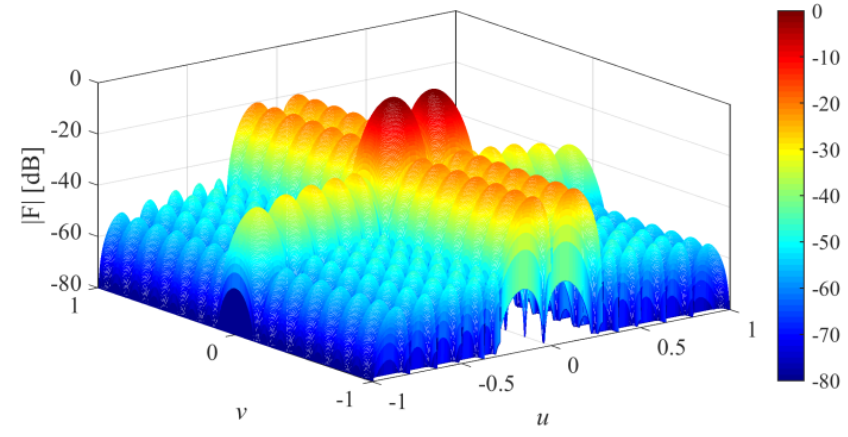

Fig. 12. The synthesized results of $15 \times 10$ array with difference-type pattern.
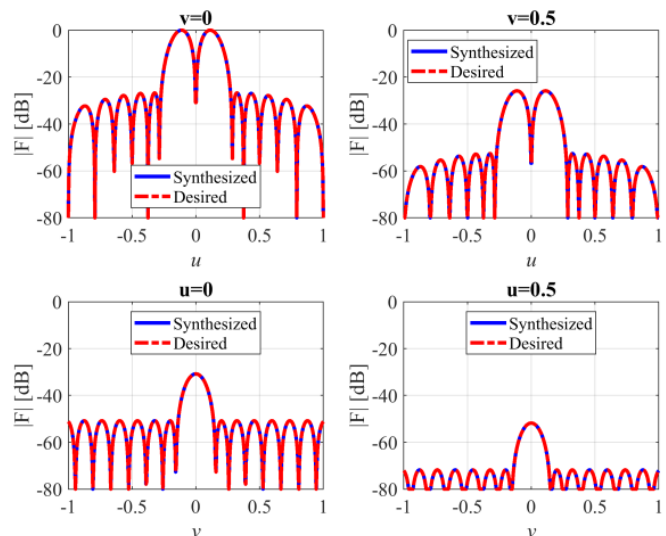

Fig. 13. Radiation pattern of the array with difference-type pattern in $\mathrm{u}-\mathrm{v}$ plane.

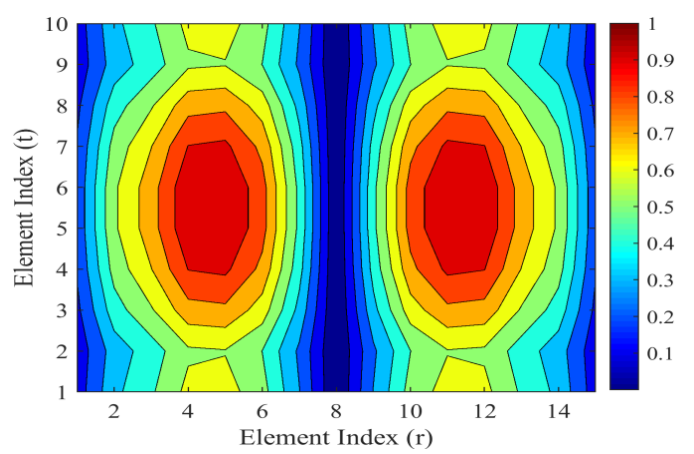

Fig. 14. The magnitude of the excitation currents of differencetype array.

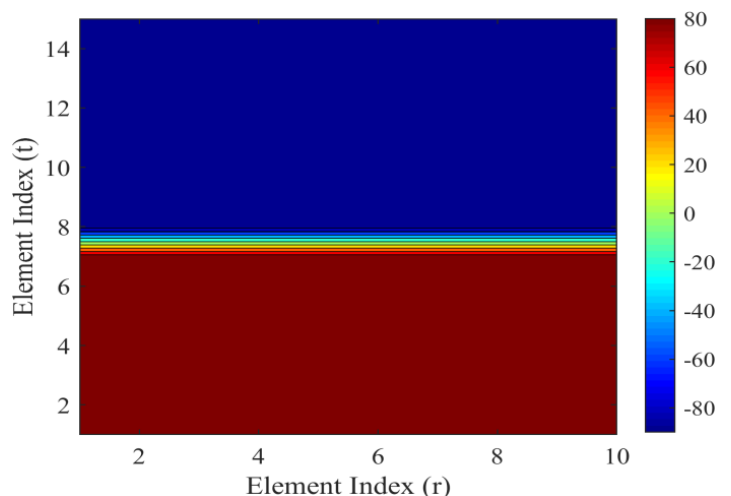

Fig. 15. The phase (in degree) of the excitation currents of difference-type array. 
depicted in Fig. 12. Also, Figure 13 shows the synthesized pattern in the $\mathrm{u}-\mathrm{v}$ plane. As seen in Fig. 13, the achieved results are extremely near to the desired one. The magnitude and phase (in degree) of the exciting coefficients are plotted in Fig. 14 and 15, respectively. As expected, an array with an asymmetrical radiation pattern has complex excitation coefficients.

\subsection{Example 5}

In the final example, a ring array with $R_{0}=2 \lambda, N=30$ is considered. The parameters of the desired array factor with Taylor distribution are $n_{\mathrm{p}}=7, \quad S L L=-20 \mathrm{~dB} \quad$ [24]. Figures 16, 17 show the obtained $3 \mathrm{D}$ and $2 \mathrm{D}$ radiation patterns, respectively. The obtained results in the $\mathrm{u}-\mathrm{v}$ plane show that the accuracy of the proposed method is high. Figure 18 displays the obtained $I_{n}$ 's versus angular position of elements in cylindrical coordinate. As expected, $I_{n}$ 's are

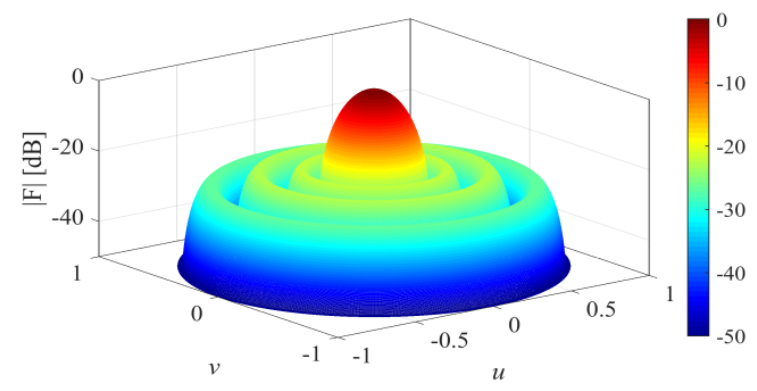

Fig. 16. The $3 \mathrm{D}$ synthesized result of ring array.
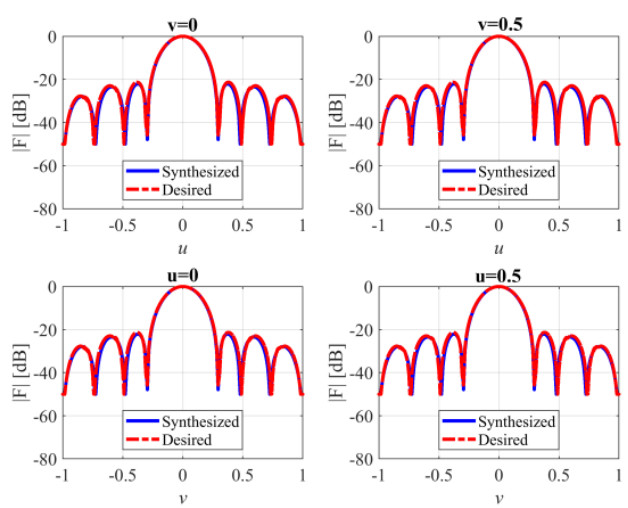

Fig. 17. Radiation pattern of ring array in $u-v$ plane.

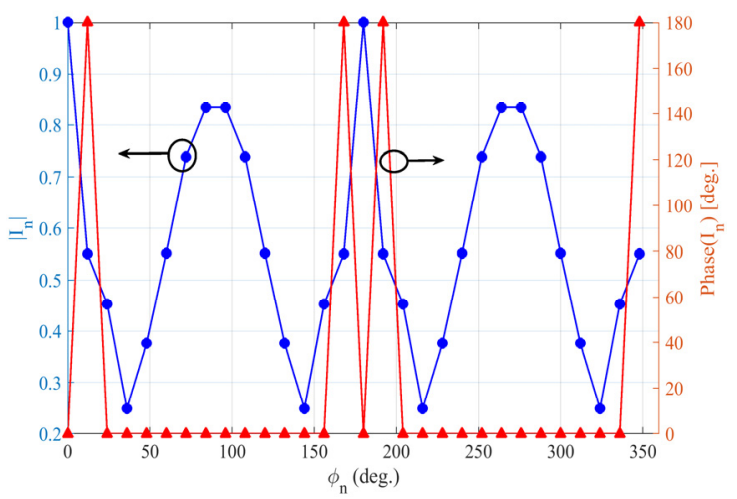

Fig. 18. The magnitude and phase of $I_{n}$ of ring array.

\begin{tabular}{|c|c|c|c|c|c|}
\hline Example \# & 1 & 2 & 3 & 4 & 5 \\
\hline MSE & $5.5 \times 10^{-5}$ & $8.9 \times 10^{-2}$ & $9.5 \times 10^{-11}$ & $9.5 \times 10^{-12}$ & $8.4 \times 10^{-5}$ \\
\hline
\end{tabular}

Tab. 1. The mean square error for all examples.

\begin{tabular}{|c|c|c|c|c|}
\hline & This work & {$[\mathbf{1 0}]$} & {$[\mathbf{1 1}]$} & {$[\mathbf{1 3}]$} \\
\hline Type & analytical & algorithm & analytical & iterative \\
\hline Complexity & medium & medium & medium & high \\
\hline Accuracy & good & good & medium & good \\
\hline
\end{tabular}

Tab. 2. The comparison of the introduced and other methods.

symmetric with respect to the ring diameter. Figure 18 also demonstrates that only the elements aligned in a symmetrical axis have non-zero phases.

Table 1 reports the computed mean square error (MSE) of the proposed method for all studied arrays. It can be seen that the MSE for all cases is acceptable. Table 2 also includes a comparison of the introduced and other proposed approaches in the literature.

\section{Conclusion}

An analytical approach for synthesizing the desired array factor of the planar and ring arrays is presented in this study. It is shown that the synthesis procedure can be simplified by defining two new parameters. To accomplish this, we use the double integral to obtain two distinct Sinc functions from a dual variables function, utilizing the sampling theory notion. Additionally, a system of linear equations is obtained and solved to determine the complex excitation coefficients of the array elements. Then, the proposed method is extended to the ring array. The performance of the proposed strategy is evaluated in the final section by presenting several examples.

\section{References}

[1] BALANIS, C. A. Antenna Theory: Analysis and Design. $4^{\text {th }}$ ed. USA: John Wiley \& Sons, 2016, ISBN: 978-1-118-642060-1

[2] ZOU, L., WANG, X. T., WANG, W., et al. Ku-band high performance monopulse microstrip array antenna based on waveguide coupling slot array feeding network, Radioengineering, 2020, vol. 29, no. 1, p. 59-66, DOI: 10.13164/re.2020.0059

[3] SHARIFI, M., BOOZARI, M., ALIJANI, M. G. H., et al. Development a new algorithm to reduce SLL of an equally spaced linear array. In The $26^{\text {th }}$ Iranian Conference on Electrical Engineering (ICEE). Mashad (Iran), 2018, p. 554-557. DOI: 10.1109/ICEE.2018.8472414

[4] HAMAD, E. K. I., ABDELAZIZ, A. Performance of a metamaterial-based $1 \times 2$ microstrip patch antenna array for wireless communications examined by characteristic mode analysis. Radioengineering, 2019, vol. 24, no. 4, p. 680-688, DOI: $10.13164 /$ re.2019.0680

[5] ALIJANI, M. G. H., NESHATI, M. H., BOOZARI, M. Side lobe level reduction of any type of linear equally spaced array using the method of convolution. Progress In Electromagnetics Research Letters, 2017, vol. 66, no. 1, p. 79-84. DOI: 10.2528/PIERL16121608 
[6] BIANCHERI-ASTIER, M., DIET, A., LE BIHAN, Y., et al. UWB Vivaldi antenna array lower band improvement for ground penetrating radar applications. Radioengineering, 2019, vol. 28, no. 1, p. 92-98. DOI: 10.13164/re.2019.0092

[7] STUTZMAN, W. L., THIELE, G. A. Antenna Theory and Design $3^{\text {rd }}$ ed. USA: John Wiley \& Sons, 2013. ISBN: 978-0-470-57664-9

[8] KHALAJ-AMIRHOSSEINI, M. Synthesis of linear and planar arrays with side lobes of individually arbitrary levels. Journal of RF and Microwave Computer-Aided Engineering, 2018, vol. 29, no. 3, p. 1-9. DOI: $10.1002 /$ mmce. 21637

[9] KEIZER, W. Large planar array thinning using iterative FFT techniques. IEEE Transactions on Antennas and Propagation, 2009, vol. 57, no. 10, p. 3359-3362. DOI: 10.1109/TAP.2009.2029382

[10] QUiJANO, J. L. A., RIGHERO, M., VECCHI, G. Sparse 2-D array placement for arbitrary pattern mask and with excitation constraints: A simple deterministic approach. IEEE Transactions on Antennas and Propagation, 2014, vol. 62, no. 4, p. 1652-1662. DOI: $10.1109 /$ TAP.2013.2288363

[11] GANESH, M., SUBHASHINI, K. R. Pattern synthesis of circular antenna array with directional element employing deterministic space tapering technique. Progress In Electromagnetics Research $B$, 2017, vol. 75, p. 41-57. DOI: 10.2528/PIERB17031603

[12] ALIJANI, M. G. H., NESHATI, M. H. Development a new technique based on least square method to synthesize the pattern of equally space linear arrays. International Journal of Engineering, 2019, vol. 32 , no. 11, p. 1620-1626. DOI: 10.5829/ije.2019.32.11b.13

[13] GONG, Y., XIAO, S., YANG, B. Z. Synthesis of sparse planar arrays with multiple patterns by the generalized matrix enhancement and matrix pencil. IEEE Transactions on Antennas and Propagation, 2021, vol. 69, no. 2, p. 869-881. DOI: 10.1109/TAP.2020.3016484

[14] IGNACIO ECHEVESTE, J., GONZALEZ DE-AZA, M. A., RUBIO, J., et al. Gradient-based aperiodic array synthesis of real arrays with uniform amplitude excitation including mutual coupling. IEEE Transactions on Antennas and Propagation, 2017, vol. 65 , no. 2 , p. 541-551. DOI: 10.1109/TAP.2016.2638359

[15] HAUPT, R. L. Antenna Arrays: A Computational Approach. $1^{\text {st }}$ ed USA: John Wiley \& Sons, 2010. ISBN: 978-0-470-40775-2

[16] HANSEN, R. C. Phased Array Antennas. $2^{\text {nd }}$ ed. USA: John Wiley \& Sons, 2009. ISBN: 978-0-470-40102-6

[17] ALIJANI, M. G. H., NESHATI, M. H. Development a new array factor synthesizing technique by pattern integration and least square method. IEEE Transactions on Antennas and Propagation, 2018, vol. 66, no. 12, p. 6869-6874. DOI: 10.1109/TAP.2018.2871715
[18] BOOZARI, M. MOHTASHAMI, V. Synthesizing a uniformly spaced array pattern using integral operator for removing progressive phase shift. International Journal of $R F$ and Microwave Computer-Aided Engineering, 2020, vol. 30, no. 8, p. 1-9. DOI: $10.1002 /$ mmce. 22238

[19] KHALAJ-AMIRHOSSEINI, M. Synthesis of planar arrays by applying transformations to linear arrays. In The $9^{\text {th }}$ Iranian International Symposium on Telecommunications (IST). Tehran (Iran), 2018, p. 39-44. DOI: 10.1109/ISTEL.2018.8661041

[20] ALIJANI, M. G. H., NESHATI, M. H. A new closed-form expression for dispersion characteristics of fundamental mode of SIW by least squares method. Applied Computational Electromagnetic Society Journal (ACES), 2015, vol. 30, no. 8, p. 930-933. ISSN: 1943-5711 (online)

[21] ALIJANI, M. G. H., NESHATI, M. H. A new non-iterative method for pattern synthesis of unequally spaced linear arrays. International Journal of $R F$ and Microwave Computer-Aided Engineering, 2019, vol. 29, no. 11, p. 1-9. DOI: 10.1002/mmce. 21921

[22] KREYSZIG, E., KREYSZIG, H., NORMINTON, E. J. Advanced Engineering Mathematics. $10^{\text {th }}$ ed. USA: John Wiley \& Sons, 2016. ISBN: 978-0-470-45836-5

[23] STRANG, G. Introduction to Linear Algebra. $5^{\text {th }}$ ed. USA: Wellesley Cambridge Press, 2016. ISBN: 978-0-9802327-7-6

[24] MAILlOUX, R. J. Phased Array Antenna Handbook. $3^{\text {th }}$ ed. UK: Artech House, 2018. ISBN: 978-1-63081-029-0

\section{About the Authors ...}

Mahdi BOOZARI was born in Mashhad, Iran, 1989. He received the M.Sc. degree in Electrical Engineering from Sharif University of Technology, Tehran, Iran, in 2015. He is interested in EM wave scattering, ray tracing and antenna arrays.

Mohammad KHALAJ-AMIRHOSSEINI was born in Tehran, Iran in 1969. He received the M.Sc. and Ph.D. degrees in Electrical Engineering from the Iran University of Science and Technology (IUST), Tehran, in 1994 and 1998, respectively. He is currently a Professor with the School of Electrical Engineering, IUST. His current research interests include EM, microwaves, antennas, radio wave propagation, and EMC. 